\title{
Effect of Vermicompost on Soil Enzyme Activity of Coastal Saline Soil in
}

\author{
Water Spinach Plantation \\ Xiao Deng ${ }^{1,2, a}$, Chun-Yuan $\mathrm{Wu}^{1,2, b^{*}}$, Qin-Fen $\mathrm{Li}^{1,2, \mathrm{c}^{*}}$, Wei $\mathrm{Li}^{1,2, \mathrm{~d}}$ \\ ${ }^{1}$ Environment and Plant Protection Institute, CATAS, Haikou, 571101, China \\ ${ }^{2}$ Danzhou Scientific Observing and Experimental Station of Agro-Environment, Ministry of \\ Agriculture, Danzhou, 571737, China \\ adx0928@foxmail.com, b wuchunyuan1981@126.com, ${ }^{\mathrm{C}}$ qinfenli2005@163.com, \\ d530243942@qq.com, * Corresponding author
}

\section{Keywords: Vermicompost, Coastal saline soil, Soil enzyme activity, Water spinach}

Abstract. Using pot experiment to study the effect of vermicopost on the activities of soil urease, acid invertase, acid phosphatase and catalase of coastal saline soil in water spinach plantation under conditions without chemical fertilizer utilization. Six treatments were designed by mixing vermicompost and saline soil in different volume ratios of 0:20, 2:20, 3:20, 4:20, 5:20 and 6:20. The results showed that adding vermicompost could significantly increase the activities of four kinds of soil enzyme in water spinach plantation. And the activities of urease, acid invertase, acid phosphatase and catalase were increased by $245.9 \% \sim 378.7 \%, 30.6 \% \sim 156.8 \%, 3.6 \% \sim 21.7 \%$ and $36.3 \% \sim 147.7 \%$, respectively. The promoting effect of different treatments on soil enzyme activity showed different rules. The activities of urease, acid invertase, acid phosphatase and catalase were characterized by $4: 20>5: 20>6: 20>3: 20>2: 20,4: 20>5: 20>3: 20>6: 20>2: 20,5: 20>4: 20>$ $3: 20>2: 20>6: 20$ and $6: 20>5: 20>4: 20>3: 20>2: 20$, respectively. This indicated that the improving effect of vermicopost on the coastal saline soil was the best in the treatments with volume ratios of 4:20 5:20.

\section{Introduction}

China saline soil area is very large and widely distributed, especially in the coastal areas, the problem of land salinization is very serious [1]. High soil salinity is a key factor restricting land reclamation in coastal areas [2]. At present, it is generally believed that the biological measures are the most effective ways to improve saline soil in all kinds of technical measures. Applying organic fertilizer to improve soil salinization, on the one hand, which can change the soil physical properties, improve the crumb structure and increase the output of crops. On the other hand, the application of organic manure can increase the activity and quantity of soil microbial, promote the migration and transformation of salts in the soil and reduce salt concentration [3]. Vermicopost not only has good crumb structure, but also contains rich microorganisms and biological active substances, etc. Vermicopost can also increase the content of soil humus after plowing soil, and play to the role is better than general organic fertilizers on soil improvement [4]. Soil enzyme is one of the most active parts of soil components. It is a biological catalyst, and is involved in the whole process of soil formation, growth and soil fertility. Soil enzyme is an important index for evaluation of soil fertility, and can reflect the status of soil quality and the ecological environment situation [5]. Therefore, the activities of soil urease, acid invertase, catalase and acid phosphatase were chosen as evaluation indexes to study the effects of vermicopost on coastal saline soil, which purpose was to 
provide theoretical basis for improvement of saline soil.

\section{Materials and methods}

The tested soils were taken in coastal saline land of LuoDou farm (N 1959'28.4", E 110'37'2.3") of WenChang City in Hainan Province. And the concentration of soil chlorine ion was $2816 \mathrm{mg} \mathrm{kg}^{-1}$. Soil samples were taken from 0 20 cm tillage layer soil by applying five point sampling method. Samples were stored in a plastic bag and taken to the laboratory on the same day, the plant roots and stones were removed, and samples were passed through a $2 \mathrm{~mm}$ sieve after air drying. The tested vermicopost was from Danzhou agricultural production base of Hainan University. The tested plant was water spinach. The pot experiment was conducted in the greenhouse of Danzhou experimental base of Chinese Academy of Tropical Agricultural Sciences from August to September in 2016. Six treatments were designed by mixing vermicompost and coastal saline soil in different volume ratios of 0:20, 2:20, 3:20, 4:20, 5:20 and 6:20. Each treatment was repeated 3 times. The cultivation conditions and management methods of each pot was the same. All vermicompost and air drying soil were directly mixed for planting water spinach under conditions without chemical fertilizer utilization, and watered one time a day. After seeds sprouting, 7 plants with the same growth status were remained to observe in each pot. The potted soil samples were collected after the harvest of water spinach, and passed through a 40 mesh sieve after air drying to analyze the activities of soil urease, acid invertase, acid phosphatase and catalase. All kinds of soil enzyme activity were used Kit (Suzhou Branch Ming Biotechnology Co., Ltd., www.cominbio.com) to analyze.

\section{Results and Discussion}

Urease is closely related to the transformation of nitrogen in the soil, and can reflect part of soil productivity. Soil urease activity is used to characterize the nitrogen status of soil [6]. Fig.1 showed that the soil urease activity of coastal saline soil increased $245.9 \% \sim 378.7 \%$ after adding vermicompost in water spinach plantation under conditions without chemical fertilizer utilization. And the soil urease activity increased with increasing of adding ratio of vermicompost in the treatments with volume ratios of 2:20 4:20, while decreased with increasing of adding ratio of vermicompost in the treatments with volume ratios of 4:20 6:20. This may be due to the excessive amount of vermicompost leads to excessive soil nutrients and soil microbial activities, which results in the decrease of soil urease activity. The promoting effect of different treatments on soil urease activity of coastal saline soil was 4:20 $>5: 20>6: 20>3: 20>2: 20>0: 20$.

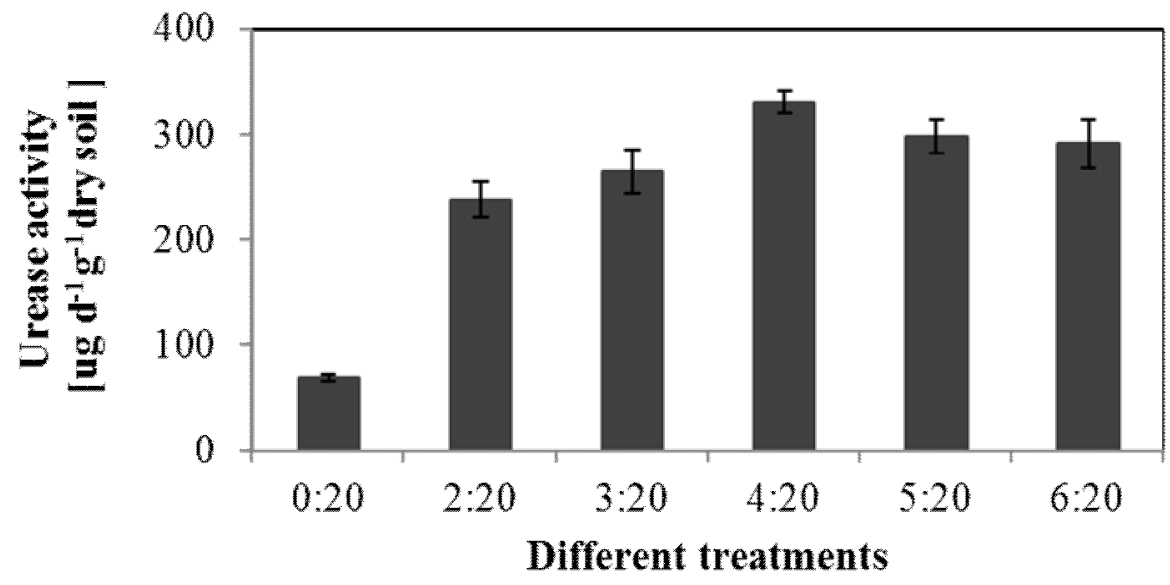

Fig. 1 Effect of vermicompost on soil urease of coastal saline soil 
Soil invertase can break down sucrose which can't be directly absorbed by plants into glucose and fructose. It plays an important role in increasing soluble nutrients in soil [7]. Soil invertase activity is often used to characterize soil maturation and fertility. Fig.2 showed that the soil acid invertase activity of coastal saline soil increased 30.6\% 156.8\% after adding vermicompost in water spinach plantation. And the soil acid invertase activity increased with increasing of adding ratio of vermicompost in the treatments with volume ratios of 2:20 4:20, while decreased with increasing of adding ratio of vermicompost in the treatments with volume ratios of 4:20 6:20. The law was consistent with soil urease. The promoting effect of different treatments on soil acid invertase activity of coastal saline soil was 4:20 > 5:20>3:20>6:20>2:20>0:20.

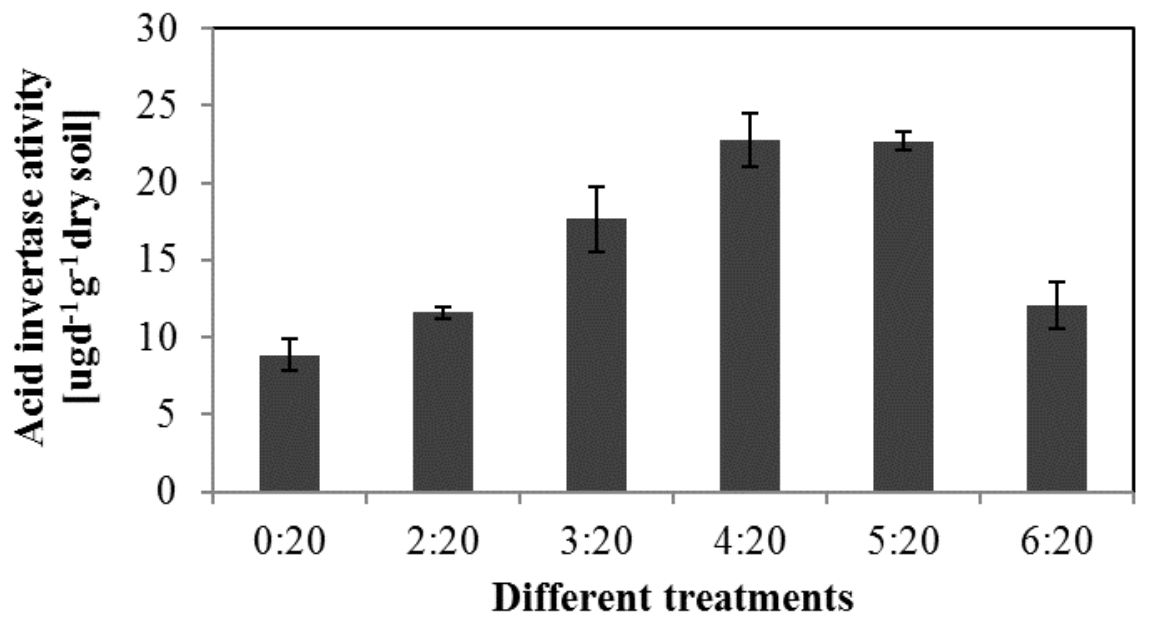

Fig. 2 Effect of vermicompost on soil acid invertase of coastal saline soil

Phosphatase can decompose organic phosphorus compounds and provide effective phosphorus for plant growth. The soil phosphatase activity can be used to characterize the soil fertility status, especially the status of phosphorus [8]. Fig.3 showed that the soil acid phosphatase activity of coastal saline soil increased 3.6\% 21.7\% after adding vermicompost in water spinach plantation. And the soil acid phosphatase activity increased with increasing of adding ratio of vermicompost in the treatments with volume ratios of 2:20 5:20, while decreased with increasing of adding ratio of vermicompost in the treatments with volume ratios of 5:20 6:20. The promoting effect of different treatments on soil acid phosphatase activity of coastal saline soil was 5:20 > 4:20> 3:20> 2:20> $6: 20>0: 20$.

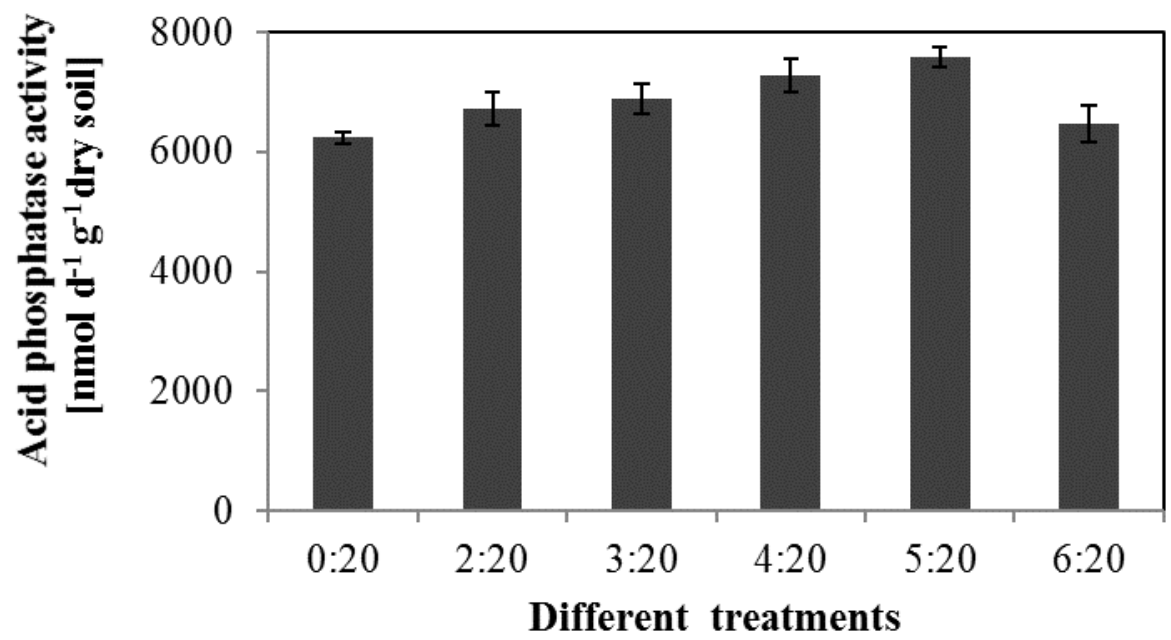

Fig. 3 Effect of vermicompost on soil acid phosphatase of coastal saline soil 
The catalase activity, which is related to soil respiration and soil microbial activity, can decompose the harmful hydrogen peroxide to water and oxygen during respiration, and reduce the harm of hydrogen peroxide to plants [9]. Fig.4 showed that the soil catalase activity of coastal saline soil increased $36.3 \% \sim 147.7 \%$ after adding vermicompost in water spinach plantation. And the soil catalase activity increased with increasing of adding ratio of vermicompost in the treatments with volume ratios of 2:20 6:20. The promoting effect of different treatments on soil catalase activity of coastal saline soil was $6: 20>5: 20>4: 20>3: 20>2: 20>0: 20$.

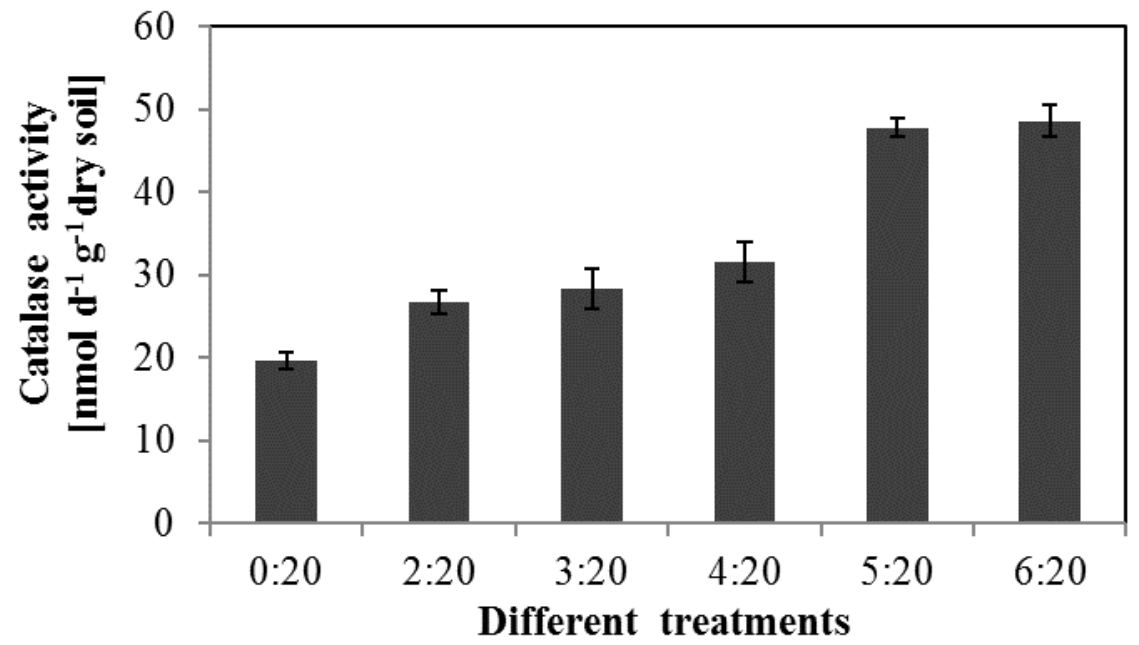

Fig. 4 Effect of vermicompost on soil catalase of coastal saline soil

\section{Conclusions}

Adding vermicompost could significantly increase the activities of urease, acid invertase, acid phosphatase and catalase of the coastal saline soil in water spinach plantation. The promoting effect of different treatments on soil enzyme activity of coastal saline soil showed different rules. And the improving effect of vermicopost on the coastal saline soil was the best in the treatments with volume ratios of 4:20 5:20.

\section{Acknowledgements}

This work was financially supported by the key research and development plan of Hainan Province (ZDYF2016149).

\section{References}

[1] Y. C. Wang, D. X. Wang. Transactions of the CSAE, Vol. 28(2012), p. 151-158.

[2] Y. Y. Wu, R. C. Liu, Y. G. Zhao. Environ Geol, Vol.57 (2009), p. 1501-1508.

[3] L. Tao, G. X. Chu, T. Liu, C. Tang, J. H. Li, Y. C. Liang. Acta Ecologica Sinica, Vol. 34(2014), p.6137-6146.

[4] Z. Y. Lu, Y. L. Ma. Chinese Agr. Sci. Bulletin, Vol. 21(2005), p. 236-240.

[5] Y. Xu, C. H. Xiang, X. W. Li. J Sichuan Forestry Sci. and Tech., Vol. 31(2010), p. 14-20.

[6] W. Q. Yang, L. Y. Wang. Scientia Silvae Sinicae, Vol. 40 (2004), p. 152-159.

[7] L. Gianfreda, F. Sanninino, A. Violante. Soil Biol. Biochem., Vol. 27 (1995), p. 1201-1208.

[8] S. Kromer, D. M. Green. Soil Biol. Biochem., Vol. 32 (2000), p.179-188.

[9] X.G. Lin. in Beijing: Higher Education Press (2010). 